\title{
Alzheimer: Cognitive, Sensory Stimulation and Relaxation, Meditation and Hypnosis through a Smart Skirting Board
}

\section{Joaquim Parra Marujo}

Transpersonal Psychology and Gerontology Research Unit, Portugal.

jmarujo@jmarujo.com

*Corresponding Author: Joaquim Parra Marujo, Transpersonal Psychology and Gerontology Research Unit, Portugal.

\section{Abstract}

Alzheimer's disease is the most common cause of dementia in old people. We used the Smart Skirting Board ${ }^{\circledR}$ $\left(S S B^{\circledR}\right)$ that integrates, among other things, a Snoezelen system, a laser system, music, video and aromas for multisensory and cognitive stimulation. The $S S B^{\circledR}$ allows a non-pharmacological intervention, and the brain stimulation is achieved, improving the elderly person's attention span, memory, mood, verbal skills and concentration and helping them to relax and offering an atmosphere of security and mental and physical relaxation. Based on a study that was conducted, the smart skirting board as a therapeutic tool, contributed significantly to the improvement of the quality of life of the elderly with psychomotor deficits and particularly those showing signs of Alzheimer's.

\section{INTRODUCTION}

Dementia is one of the biggest public health issues in this century. According to the results of the "European Collaboration on Dementia - Eurocode" Project conducted by Alzheimer Europe there are currently 7.3 million European citizens suffering from one or various forms of dementia [1]. One in every 20 people over the age of 65 has Alzheimer's disease (AD), the most common cause of dementia [2]. Experts predict that this number double in Western Europe and treble in Eastern Europe by 2040 [3]. Every year, 1.4 million European citizens develop dementia, which means that every seven seconds a new case is diagnosed. In Portugal there are 160000 people with dementia [4], of which 90000 have AD [5] diagnosed. But the real number of people affected by dementia is much larger than these statistics suggest.

Due to the increasing number of early diagnoses of people with dementia, there is a growing demand for treatments, different from the conventional, that may improve cognitive functions, apart from a possible delay of the disease. Consequently, there is a need for non-pharmacological interventions such as the $\mathrm{SSB}^{\circledR}$ a gerontodesign [6] and gerontotechnology artifact - integrating a Snoezelen system, a laser system, music, video and aromas for multisensory and cognitive stimulation. Through the Snoezelen system along with other systems introduced into the skirting board, brain stimulation is achieved, improving the elderly person's attention span, memory, mood, verbal skills and concentration and helping them to relax and offering an atmosphere of security and mental and physical relaxation

\section{AiM \& OBJECTIVES}

With this work we aim to: validate the importance of smart skirting board (SSB) to create a holistic therapeutic space; stimulate cognitively older people with Alzheimer's and reconnect older people to spirituality through relaxation, meditation and hypnosis.

\section{METHODS}

This research was conducted at the residence of São Pedro in Malveira, Portugal and included forty-five elderly people with ages between 75 and 95 years old. The diagnosis of probable Alzheimer dementia was established following the neurological criteria. For this study we used a room equipped with the SSB® (an 
Alzheimer: Cognitive, Sensory Stimulation and Relaxation, Meditation and Hypnosis through a Smart Skirting Board

invention of J Marujo and V Fernandes) as a holistic therapeutic tool that had integrated a Snoezelen system; a laser system; music; video; objects with different smells, textures and colors and different aromas (orange, lemon, strawberry, vanilla, chocolate, etc.). The sessions with 50 minutes per week, carried out between May 2016 and August 2017, were divided into two categories: One to do cognitive stimulation and another to reconnect older people to spirituality. The results of cognitive stimulation's sessions were registered in a battery of neuropsychological tests to assess cognitive function. The sessions to reconnect older people to spirituality through meditation and relaxation techniques have been proven with a gerontotranscendence's questionnaire.

\section{RESULTS}

In this holistic therapeutic space created with SSB we stimulated the primary senses such as vision, hearing, touch, taste, smell, providing feelings of enjoyment, promoting cognitive development and meeting with spirituality (Tao). We verified that all participants with Alzheimer's dementia had significant improvements and those who wanted to participate in sessions to reconnecting with spirituality, also had reconnected to religiosity.

The $\mathrm{SSB}^{\circledR}$ was created with the following objectives: 1 - To facilitate the mobility and safety of elderly people who may (or may not) be in a wheelchair and who reveal (or not) depressive disorders or dementia; 2 - Provide safety and well being to the elderly with cognitive deficits, particularly those suffering with Alzheimer's; 3 - Encourage cognitive modifiability through the cognitive and multisensory stimulation of elderly with dementia; 4 - To stimulate cognitive and spiritual functions through meditation and hypnosis.

At the end of this preliminary study we verified that all the elderly people showed a big improvement. We verified that the $\mathrm{SSB}^{\circledR}$ stimulating the senses, it helps reconnect the dementia patient to the world they left behind; by providing an enjoyable experience, it helps to reduce antisocial behavior and provides an environment that encourages meaningful relationships among staff and patients, promoting relaxation, and reducing psychological stress and staff burnout. Other benefits include relaxed patients, increased sense of happiness and interest, reduced sadness and fear, an increase in sociability and level of interest, and a decrease in disruptive behaviors and levels of anxiety.

\section{CoNCLUSIONS}

The SSB allows you to create a therapeutic space that stirs up feelings of joy, pleasure, well-being and happiness. At the same time can also be used as a tool for a learning experience, cognitive stimulation in the treatment of pain, anxiety, depression, stress, relaxation and recreation. A critical analysis of this study shows the importance of neuropsychological rehabilitation programs through a holistic therapeutic space created by the SSB in stimulating cognitive abilities. Through relaxation techniques, meditation and hypnosis is possible reconnect older people to spirituality providing them welfare and happiness.

\section{REFERENCES}

[1] In: http://www.alzheimer-europe.org/Policyin-Practice2/EU-Presidencies/2009-SwedishEuropean-Presidency/Swedish-PresidencyConference/(language)/eng-GB (Accessed on September 16, 2013).

[2] Warner J, Butler R. Alzheimer's disease. Clin Evidence. 3: 419-425, 2000.

[3] Ferri CP et al. Global prevalence of dementia: a Delphi consensus study. Lancet. 336: 2112-17, 2005.

[4] Draft National Dementia Plan is submitted to the Portuguese government In: http://www. alzheimer-europe.org/News/Policy-Watch/Friday23-August-2013-Draft-National-Dementia-Planis-submitted-to-the-Portuguese-government/ (language)/eng-GB (Accessed on September 16, 2013).

[5] Portugal - National Dementia Plans In: http:// www.alzheimer-europe.org/Policy-in-Practice2/ National-Dementia-Plans/Portugal/MP-TeresaCaeiro-interview/(language)/eng-GB (Accessed on September 16, 2013).

[6] Marujo JP. Gerontodesign: the trademark of the design, the ergonomic design, the brand or noname brands? Working papers: "Senses and sensibility in technology": linking tradition to innovation through design. pp. 290-296, Lisbon, IADE, 2003. 
Alzheimer: Cognitive, Sensory Stimulation and Relaxation, Meditation and Hypnosis through a Smart Skirting Board

\section{Author's Biography}

Joaquim Parra Marujo is a Gerontologist, Coordinate Professor of Gerontology and Transpersonal Psychology in Research Unit of Transpersonal Psychology and Gerontology. He is also a Visiting Professor of Transpersonal Psychology in Instituto Superior de Psicologia Aplicada. He has PhD in Social and Cultural Anthropology, Master in Clinical Mental Health, Master in Sociology, Economics and Politics of the Lusophone Space. He is also a member of the following societies like Spanish Society of Geriatrics and Gerontology, Portuguese Society of Geography, Portuguese Society of Anthropology and Ethnology, Portuguese Association of Psycho-gerontology, Network of Latin American Anthropologists and International Association of Counselors and Therapists. He has more than 40 articles published in journals and books in the specialty of gerontology, psychology and design and he gave more than 150 submissions and interviews at conferences, seminars, TV, radio fields of gerontology, geriatrics, transpersonal psychology and design.

Citation: Joaquim Parra Marujo. Alzheimer: Cognitive, Sensory Stimulation and Relaxation, Meditation and Hypnosis through a Smart Skirting Board. Open Journal of Geriatrics. 2019; 2(1): 26-28.

Copyright: (C) 2019 Joaquim Parra Marujo. This is an open access article distributed under the Creative Commons Attribution License, which permits unrestricted use, distribution, and reproduction in any medium, provided the original work is properly cited. 